\title{
Multimodal pipe-climbing robot with origami clutches and soft modular legs
}

\author{
Yongkang Jiang ${ }^{1,2}$, Diansheng Chen ${ }^{1 *}$, Hongying Zhang ${ }^{2}$, Frédéric Giraud ${ }^{2}$ and Jamie \\ Paik ${ }^{*}$ \\ ${ }^{1}$ Institute of Robotics, Beihang University, Beijing, China \\ ${ }^{2}$ Reconfigurable Robotics Laboratory, École Polytechnique Fédérale de Lausanne (EPFL), Lausanne, \\ Switzerland
}

E-mails: connarjiang@gmail.com, chends@163.com*, hongying.zhang@epfl.ch, frederic.giraud@epfl.ch and jamie.paik@epfl.ch*

* Authors to whom any correspondence should be addressed

\begin{abstract}
In nature, climbing trees and pipes of varying diameters or even navigating inside of hollow pipes and tree holes is easy for some climbing animals and insects. However, today's pipeclimbing robots, which are important for automatically conducting periodic inspections and maintenance of pipelines to save time and keep humans away from hazardous environments, are designed mainly for a specific task, limiting their adaptability to different working scenarios and further implementation in real-life. In this paper, we propose a pipe-climbing robot with a soft linear actuator for bioinspired propulsion, two origami clutches to realize multi-degrees-of-freedom (DoF) motion and two pairs of soft modular legs for multimodal climbing. Design, modeling and experimental validation of the origami clutch are introduced in detail. Preliminary experimental results show that we can achieve a stroke of up to $289.6 \%$ and a maximum 45 degrees bending angle on the soft linear actuator by regulating the air pressure inside the soft actuator and origami clutches. Additionally, by choosing the leg-type, three climbing modes, including out-pipe versatile mode, out-pipe high-force mode and inpipe mode can be realized for particular working scenarios. A prototype climbing robot demonstrates that in out-pipe versatile mode, the robot can climb on the exterior of pipes made of various materials including PVC, rubber and metal with diameters ranging from 105 to $117 \mathrm{~mm}$. In the out-pipe high-force mode, the climber can navigate along a specific pipe carrying maximum $675 \mathrm{~g}$ external load at the top or $200 \mathrm{~g}$ hanging from the bottom, as well as keeping functional without failure under static loads as high as $1968 \mathrm{~g}$. In the in-pipe mode, the robot is able to travel inside pipes. This research might bridge the design gap between in-pipe and out-pipe climbing robots while offering an alternative option for soft robots to execute multi-DoF motion.
\end{abstract}

Keywords: pipe-climbing robot, soft robotics, origami clutch, modular design

\section{Introduction}

Pipelines are widely utilized in many industrial environments as well as in our daily lives, such as petrochemical plants, water/electricity transportation systems and heat exchange networks. At present, most of the periodic safety inspections and maintenance of pipelines to prevent critical leakage accidents are performed manually, which is quite time-consuming and may be dangerous for humans exposed to the hazardous conditions.

To address this threat, several pipe-climbing robots have been developed recently that can perform inspection and maintenance on pipelines remotely [1,2]. Existing climbing robots can be classified into major categories according to locomotion principles, including legged type $[3,4]$, wheeled/tracked type [5-8] and inchworm type [9-11]. Of note 

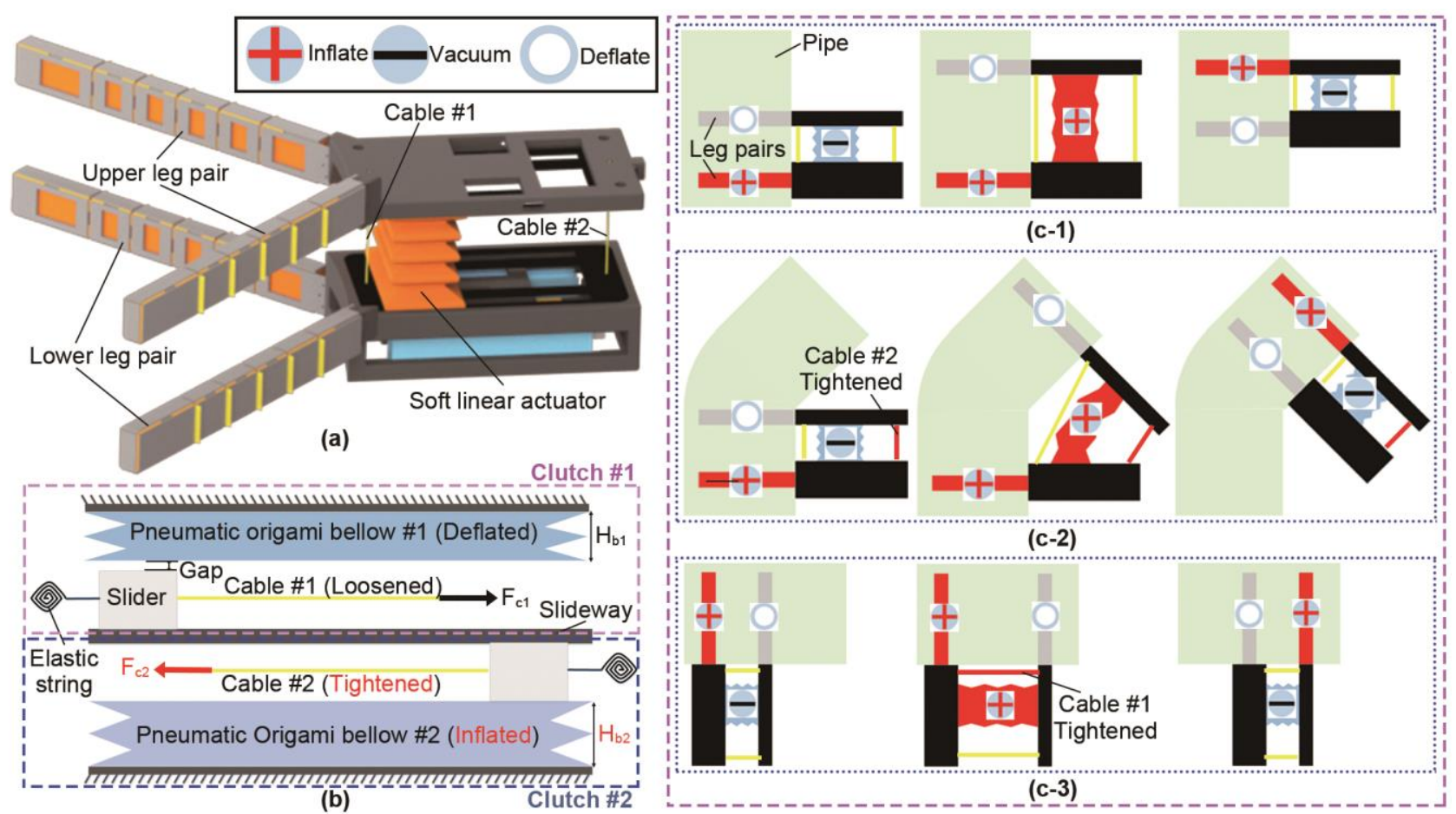

Figure 1. (a) Schematic of a multimodal pipe climbing robot consisting of a soft linear actuator, two pneumatic origami clutches and two pairs of legs. (b) Working principle of the pneumatic origami clutches. (c) Locomotion of the pipe-climbing robot is realized by periodic actuation of the soft linear actuator and legs and then selectively regulating the pulling force on the cables by adjusting the air pressure inside the corresponding origami clutches: (c-1) climbing up a vertical pipe with all cables loosened (clutches deflated); (c-2) navigating the exterior of a pipe with turns by selectively tightening cable \#2 (clutch \#2 inflated, clutch \#1 deflated) to adjust the bending angles of the soft linear actuator; (c-3) traveling through a horizontal pipe by selectively tightening cable \#1 (clutch \#1 inflated, clutch \#2 deflated) to maintain the movement direction of the climber and avoiding failure induced by its own weight or external load.

most of the existing pipe-climbing robots are based on rigid frameworks and are driven by electric motors, which have advantages for multi-DoF motion and precise control. However, their usually cumbersome bodies might be a limitation. With the rapid development of soft robots, an emerging research field that has gained increasing attention world-wide [12], soft pipe-climbing robots have shown tremendous merit because they tend to be light-weight, lowcost and inherently compliant [13,14]. Nevertheless, the ability to realize multi-DoF motion remains a challenge for existing soft robots. Nowadays, available solutions include utilizing multiple chambers $[15,16]$ or introducing stiffnesschangeable smart materials $[17,18]$. However, for the multichamber soft pipe climbing robots $[19,20]$, failure might occur when they navigate along horizontal or inclined pipes carrying external loads because their maximum mechanical stiffness is limited by the inherent low stiffness of soft materials. Besides, drawbacks also exist for the smart materials, including the high hysteresis of jamming mechanism [21,22] and the long cooling times for phase transition-based materials to recover to the initial high- stiffness state [23]. Hence, we need a new method for soft pipe-climbing robots to carry out multi-DoF motion for dealing with pipelines with turns.

Furthermore, the existing pipe-climbing robots can be roughly divided into two main categories based on their working scenarios: in-pipe climbing robots that navigate inside pipes and the out-pipe counterparts, climbing along the outside [24]. In-pipe climbing robots navigating the inside of pipes bypass obstacles usually found outside of pipes such as valves, fixtures and flanges. Besides, for underground pipelines where there is not enough space for out-pipe climbing robots, in-pipe climbers are usually the only available choice. Nevertheless, whole pipelines have to be shut down and any fluids inside removed before deployment of climbers. Their counterparts, out-pipe climbing robots can function well hanging onto pipelines without complicated preparatory procedure and regardless of the contents inside the pipes. However, most existing pipe-climbing robots are designed for a specific task inside or outside of pipelines [1], which limits their adaptability and versatility. Hence, developing a pipe-climbing robot that can perform well both inside and outside pipelines may bridge the design gap between in-pipe and out-pipe climbing robots.

In this paper, we develop a multimodal pipe-climbing robot with origami clutches and soft modular legs. First, we describe the structure and working principle of the pipe-climbing robot. Then, we illustrate the analytical models concerning the clutch in detail and compare them with finite element analysis (FEA) 
and the experimental results. We go on to propose three types of soft modular legs for multimodal climbing of the robot and enhance grasping force of the legs by managing to avoid the insufficient contact between the soft actuators and pipes induced by excessive deformation of the soft structures with overlarge input air pressure, as mentioned in $[25,26]$. Finally, we develop a preliminary prototype of the pipe-climbing robot and evaluate its functionality with each leg-mode in three different working scenarios. The experimental results showed that by selectively adjusting the input air pressure inside the origami clutches and replacing the different leg modules, three modes of climbing in various working scenarios can be achieved, including (1) out-pipe versatile mode with two pairs of normally open legs to travel along the exterior of pipelines with bends, varying diameters and different materials; (2) outpipe high-force mode with normally closed legs to climb up pipes of a set diameter with limited loads and remain functional under high static load; (3) in-pipe mode to navigate inside pipes.

The contributions of this research can be highlighted as:

- Design, modeling and validation of control parameters of a novel origami clutch for multi-DoF bioinspired propulsion;

- Modular design of soft-rigid hybrid legs for multimodal climbing and gripping force enhancement of mobile robot;

- A functional prototype of a multi-legged pipe-climbing robot and performance evaluation in three working scenarios.

\section{Climbing robot design}

In nature, some climbing animals and insects are capable of embracing pipes or trees with their limbs, forming a ring-like shape that increases holding force by enlarging the contact surface. Then, by periodic body propulsion and alternatively holding or relaxing their limbs, the climbing animals and insects climb different pipes or trees easily.

Similar to these natural counterparts, the proposed multilegged pipe-climbing robot consists of a soft body and two pairs of soft modular legs as illustrated in Figure 1(a). Below we introduce the system overview of the robot, followed by a description of the structure and working principles of the robot body with origami clutches and soft modular legs.

\subsection{System overview}

As with the extreme importance of the body structure and strong limbs of climbing animals, the main challenges in designing pipe-climbing robots include a versatile body for multi-DoF propulsion and large-stroke movement, and functional legs to exert a high-enough holding force in numerous working scenarios.

For the body, a bellow-like soft linear actuator was adopted since it has advantage of achieving large stroke when vacuumed and inflated [27]. A hard question for this design is the way to regulate movement direction of the soft linear actuator for multi-DoF motion when dealing with pipes with turns. As mentioned above, existing solutions for soft structures to achieve multi-DoF motion are limited by their own disadvantages. To address this and avoid using other actuation sources that could make the system cumbersome, a novel pneumatic origami clutch is proposed in this work. Schematic of the main components of the pneumatic origami clutches is given in Figure 1(b). Each clutch contains a pneumatic origami bellow whose height varies according to the input air pressure, a 3D-printed slider with one end connected to the upper part of the climber via a Kevlar cable and the other end attached to an elastic spring for returning to the initial position. Additionally, a shared slideway is mounted between the sliders so that they can travel through it.

The main feature of the working principle of the clutch to regulate the pulling force on the cable is: when the origami bellow is deflated, there will always be a tiny gap between the slider and the bellow, which means that the slider can move easily along the slideway without much impedance and the pulling force needed to move the slider is relatively small; once the origami bellow is inflated, the bellow will contact the slider and press it towards the slideway, significantly increasing the pulling force on the cable that can be further regulated by adjusting the input air pressure inside the bellow. In should be noted that with the help of friction tape, the friction coefficient on the interface between the slider and the bellow is much higher than that between the slider and slideway (further calibration can be found in the next section). With this design, the movement direction of the soft linear actuator can be regulated by selectively tightening or loosening the cables by modulating the input air pressure inside the origami clutches.

For the legs, the biggest challenge is their adaptability to several working scenarios including in-pipe and out-pipe climbing and a large enough holding force to avoid failure due to the robot's weight. For the adaptability, three types of replaceable legs based on modular design are proposed in this work and we show one of them in this section (see Figure 1(a)) for simplification. When inflated, this pair of legs will bend towards a pipe to hold the upper or lower part of the climber, similar to the function of limbs of primates when climbing. For the holding force, a soft-rigid hybrid design is proposed and will be introduced in detail in the following section.

A typical climbing cycle of the proposed robot on a vertical pipe can be achieved by periodic actuation of the soft linear actuator and the two pairs of legs as depicted in Figure 1(c-1). Inflation and vacuum of the soft linear actuator will induce body propulsion and at the same time inflating or deflating two pairs of legs will allow them to hold the upper or lower part of the robot against the pipe. Likewise, the elongation of the soft linear actuator could also result in a bending motion of the 


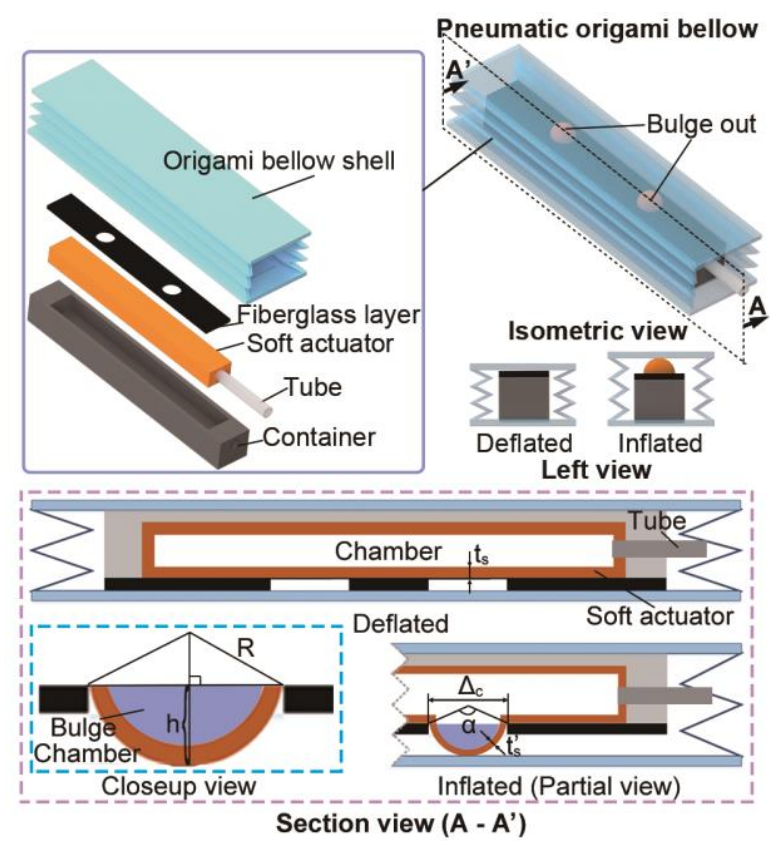

Figure 2. Main components of the pneumatic origami bellow and parametric description of the deformation of the soft rectangular actuator inside the origami bellow. Isometric view is upward to better show the location of silicone bulges. Section view (A-A') is taken along the longitudinal direction of isometric view and then inverted downward to show how silicone bulges push down the bottom plate of the origami bellow shell in reality. Closeup view of one of the silicone bulges is given to better depict geometric parameters.

robot as shown in Figure 1(c-2), when cable \#2 is tightened by increasing the air pressure inside origami clutch \#2 so that the climber can travel through pipes with turns. Similarly, the climbing robot could also travel through a horizontal pipe by selectively tightening cable \#1 to maintain the movement direction of the climber and avoid failure induced by its own weight as illustrated in Figure 1(c-3).

\subsection{Loading on the body with origami clutches}

As a main component of the body, the pneumatic origami clutch plays an important role in realizing multi-DoF climbing by regulating the movement direction of the soft linear actuator. The most important part is the actuation method of the pneumatic origami bellow to adjust its height. According to our previous work [28], it is quite difficult to keep the bellow air-tight at relatively high air pressure and the output force is limited. Here, we propose a novel design of a pneumatic origami bellow by combining origami and soft robotic technology. As illustrated in Figure 2, the bellow consists of four main components: a light-weight origami bellow shell, a rectangular soft actuator made of Dragon Skin 30 with 10\% Silicone Thinner, a 3D-printed container to restrict expansion of the soft actuator at the lateral and bottom faces, and a laser-cut fiberglass layer with two holes that allows the silicone to bulge out to increase the height of the bellow as depicted in the Figure 2 left view. When the soft actuator is inflated, the silicone bulges will contact the origami bellow shell and push it towards the slider to enhance the pulling force on the cable as mentioned above. This design has two main advantages: higher pressure can be sustained by the soft actuator, meaning that the bellow can push the slider harder and hence achieve larger pulling force on the cable; the origami bellow insures that the silicone bulges do not contact the slider directly, which significantly increases the lifetime of the mechanism.

To quantitatively analyze the relationship between the pulling force on the cable and the input air pressure inside the soft rectangular actuator as the slider moves along the slideway, analytical models are given and detailed derivations can be found below. The theoretical models consist of two main parts: (1) the deformation analysis of the height of the silicone bulges when the applied air pressure varies; (2) deviation around the pulling force on the cable under a certain input air pressure, which indicates a certain deformation height of the silicone bulges as the displacement of the slider increases along the slideway. Assumptions are made to simplify the models: the elastic force and weight of the origami bellow as well as weight of the slider are empirically neglected as they are very small compared to the pushing force of the soft rectangular actuator according to experimental results.

First, the relationship between the deformed height of the soft rectangular actuator and the input pressure is analyzed. Note that the mechanism is totally symmetric for the two silicone bulges, dealing with either works.

Yeoh material model is adopted here according to the material tests introduced in Section 3 and the strain-energy function $u$ can be given as,

$$
u=\sum_{i=1}^{3} c_{i}\left(I_{1}-3\right)
$$

where $c_{i}$ is the material coefficients to be calibrated and $I_{1}$ is the first invariant of the Cauchy-Green strain tensor which can be expressed as,

$$
I_{1}=\lambda_{1}^{2}+\lambda_{2}^{2}+\lambda_{3}^{2}
$$

where $\lambda_{1}, \lambda_{2}, \lambda_{3}$ are the stretch of the soft material in longitudinal, circumferential and radial direction respectively. Considering the boundary conditions in this case and the inextensibility of the soft material, the stretches can be obtained as: $\lambda_{2}=\lambda_{1} ; \lambda_{3}=\lambda_{1}^{-2}$. According to virtual work principle, the energy equation can be given as,

$$
\delta U=\delta W,
$$

where $\delta U$ denotes the strain-energy stored in the soft material and $\delta W$ is the work of the air pressure when the soft actuator deforms. They can be further obtained as,

$$
\delta U=V_{s} \delta u, \delta W=P \delta V_{c},
$$

where $V_{s}$ refers to the initial volume of the silicone bulge, $P$ is the input air pressure and $V_{c}$ denotes the volume of the 


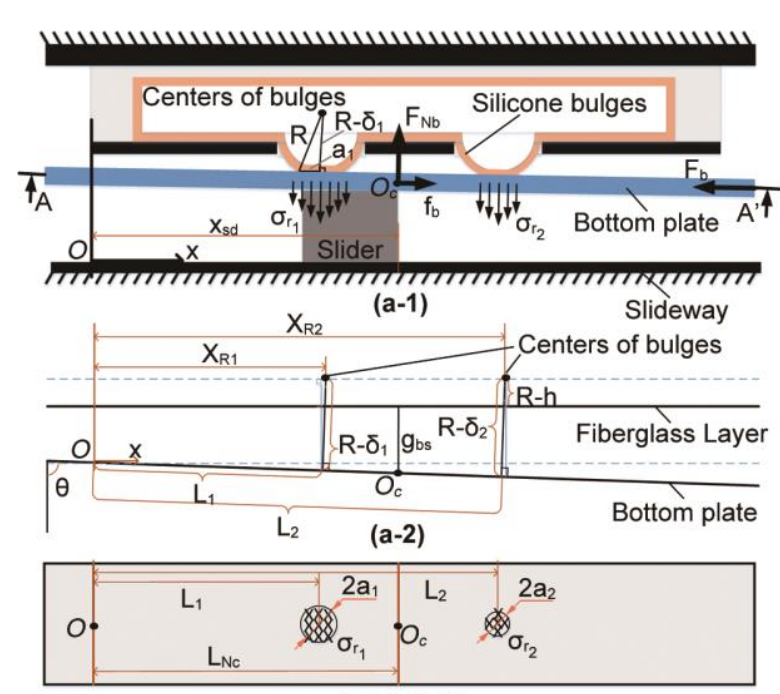

(a-3) A-A

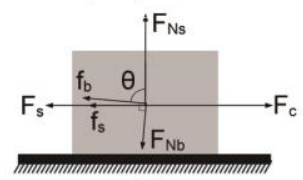

(b)

Figure 3. Description of a typical contact pattern of the silicone bulges, bottom plate of the origami bellow shell and the slider. (a-1) overview. (a-2) Detailed sketch of (a-1) showing main geometric parameters. (a-3) Section view of force analysis on the bottom plate of the bellow shell. (b) Force analysis of the slider.

chamber of the bulge as illustrated in the closeup view of Figure 2. It can be obtained that,

$$
\begin{gathered}
V_{s}=\frac{\pi t_{s} \Delta_{c}^{2}}{4}, \quad V_{c}=\frac{\pi(3 R-h) h^{2}}{3}-V_{s} \\
R=\frac{4 h^{2}+\Delta_{c}^{2}}{8 h}, \quad \lambda_{1}=\frac{\alpha R}{\Delta_{c}},
\end{gathered}
$$

where $t_{s}\left(t_{s}=1 \mathrm{~mm}\right)$ is the initial thickness of the bulge, $\Delta_{c}$ ( $\Delta c=5 \mathrm{~mm}$ ) refers to the diameter of the hole, $R$ is the radius of the deformed silicone bulge, $h$ denotes the deformed height and the angle $\alpha$ as depicted in Figure 2 can be further given as,

$$
\alpha=\left\{\begin{array}{cl}
2 \arcsin \left(\frac{\Delta_{c}}{2 R}\right), & h \leq R \\
2 \pi-2 \arcsin \left(\frac{\Delta_{c}}{2 R}\right), & h>R .
\end{array}\right.
$$

By substitution of (1), (2) and (4)-(6) into (3), it can be obtained that deformed height $h$ is a function of the input pressure $P$ as briefly concluded as,

$$
h=f(P) .
$$

Then, it is the derivation of the relationship between the pulling force on the cable $F_{c}$ and the deformed height $h$ of the silicone bulges as the slider moves along the slideway. As introduced above, when the soft rectangular actuator in the origami bellow is inflated, the silicone bulges rise up from the holes in the laser-cut layer and then contact the bottom plate of the origami bellow that will accordingly contact the slider to enhance the pulling force on the cable finally. In fact, there will be several similar contact phases as the displacement of the slider along the slideway $x_{s d}$ increases. For simplification, a typical contact pattern as shown in Figure 3 is given in this work.

As in Figure 3 (a-2), the geometrical equations can be given as,

$$
\begin{gathered}
L_{1} \sin \theta-x_{R 1}+\left(R-\delta_{1}\right) \cos \theta=0 \\
\left(R-h+g_{b s}+\left(L_{2}-L_{N c}\right) \cos \theta-\left(R-\delta_{2}\right) / \sin \theta=0\right. \\
\left(R-h+g_{b s}-\left(L_{N c}-L_{1}\right) \cos \theta-\left(R-\delta_{1}\right) \sin \theta=0,\right.
\end{gathered}
$$

where $\delta_{1}, \delta_{2}$ are the distance variation between the centers of the silicone bulges and bottom plate of the origami bellow shell respectively, $x_{R 1}=17.5 \mathrm{~mm}, x_{R 2}=42.5 \mathrm{~mm}$ denote the horizontal distances between the centers of silicone bulges and the reference point $O$ respectively, $\theta$ refers to the angle of inclination of the bottom plate and other variables can be found in Figure 3.

To obtain the contact force that the silicone bulges exert on bottom plate of the origami bellow shell, Hertz contact model [29] has been utilized and the distributed stresses $\sigma_{r_{i}}$ on the contact surface should obey Cauchy distribution as,

$$
\begin{gathered}
\sigma_{r_{i}}=\frac{2}{\pi} E^{*}\left(\frac{\delta_{i}}{R^{*}}\right)^{\frac{1}{2}} \sqrt{1-\left(\frac{r_{i}}{a_{i}}\right)^{2}}, i=1,2 \\
a_{i}=\sqrt{\delta_{i} R^{*}}, i=1,2 \\
\frac{1}{E^{*}}=\frac{1-v_{s}^{2}}{E_{s}}+\frac{1-v_{b}^{2}}{E_{b}} \\
\frac{1}{R^{*}}=\frac{1}{R}+\frac{1}{R_{b}},
\end{gathered}
$$

where $a_{i}$ denotes the radii of the contact areas, $r_{i}$ refers to the distance to the center of the contact areas, $E^{*}$ and $R^{*}$ are the effective elastic modulus and bending radius respectively, $E_{s}$ and $E_{b}$ are the elastic modulus of the silicone and the bellow respectively $\left(E_{b} \square E_{s}\right), v_{s}$ and $v_{b}$ are the Poisson's ratio of the silicone and bellow respectively. It can be given that $R^{*} \approx R$ since the bending radius of the bellow plate is almost infinite.

According to Figure 3(a-3) and 3(b), the force equilibriums of the bellow plate as well as the slider can be obtained as,

$$
\begin{gathered}
F_{c}=F_{N b}\left(\cos \theta+\mu_{b} \sin \theta+\mu_{s} \sin \theta-\mu_{s} \mu_{b} \cos \theta\right)+F_{s} \\
F_{N b}-\left(F_{N c}+\sum_{i=1}^{2} F_{i}\right)=0 \\
F_{i}=\int_{0}^{a_{i}} \sigma_{r_{i}} 2 \pi r_{i} d r_{i}=\frac{8 G_{s}}{3\left(1-v_{s}\right)} \sqrt{R \delta_{i}^{3}}, i=1,2,
\end{gathered}
$$




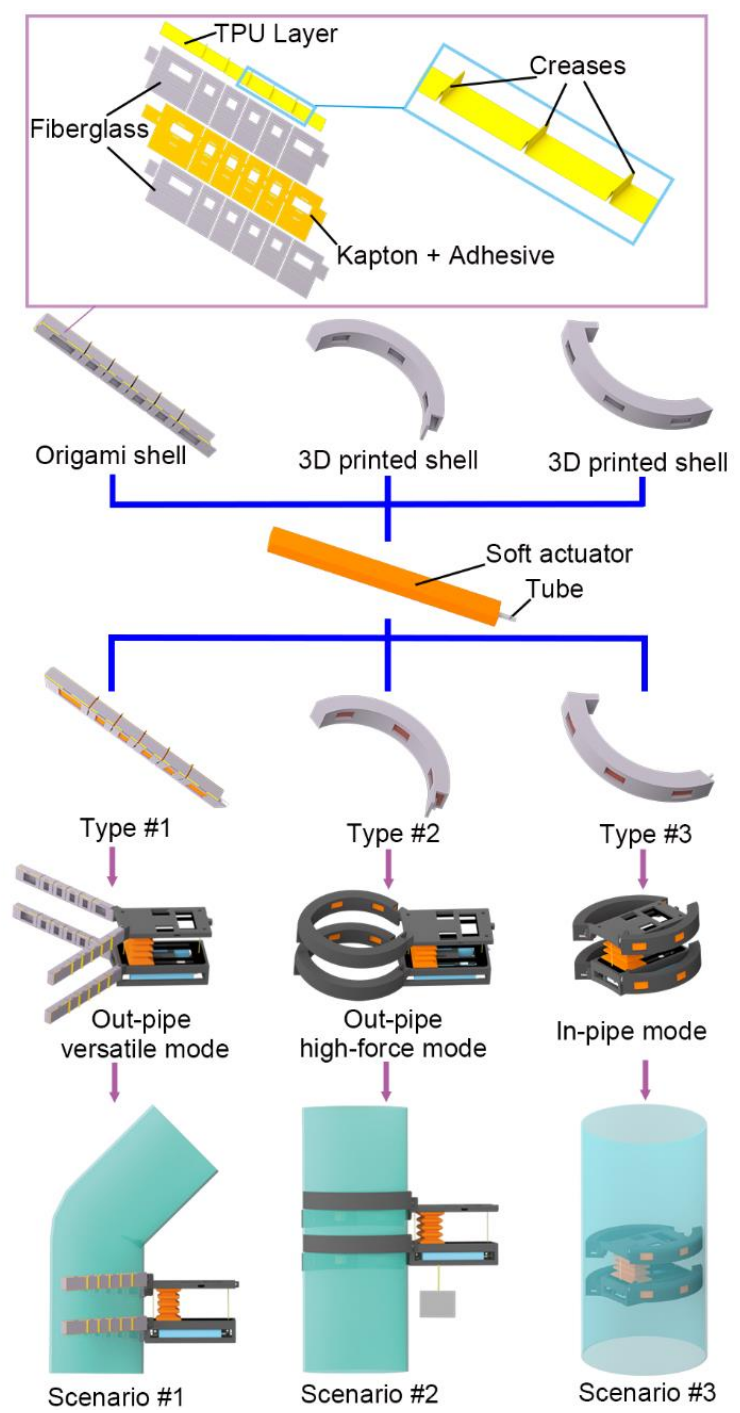

Figure 4. Structure description of the three types of legs, schematic of working modes of the pipe-climbing robot by integrating each legtype with the main body of the robot respectively and their application in three working scenarios.

where $F_{c}$ is the pulling force on the cable, $F_{N b}$ denotes the normal pushing force that the bellow plate exerts on the slider, $F_{N c}$ is the normal pushing force of the slideway, $F_{s}$ refers to the recovering force of the elastic string as in Figure $1, F_{i}$ denote resultant forces of the distributed stresses $\sigma_{r i}$ on the contact surface respectively, $G_{s}$ is the shear modulus of the silicone, $\mu_{b}$ refers to the friction coefficient between the slider and bellow plate and $\mu_{s}$ is the friction factor between the slider and the slideway. $F_{s}, G_{s}, \mu_{b}$ and $\mu_{s}$ will be experimentally calibrated in Section 3. Furthermore, the moment equilibriums of the bellow plate around the contact point $O_{c}$ as illustrated in Figure 3 can be obtained as,

$$
\begin{gathered}
M_{1}-M_{2}=0 \\
M_{1}=F_{1}\left(L_{N c}-L_{1}\right) \\
M_{2}=F_{2}\left(L_{2}-L_{N c}\right),
\end{gathered}
$$

where $M_{1}, M_{2}$ denote the torque of forces $F_{1}, F_{2}$ respectively.

For a certain deformed height of the soft actuator when the input air pressure inside the actuator is given, the theoretical relationship between the pulling force $F_{c}$ and the displacement of the slider $x_{s d}$ as it moves along the slideway can be calculated by combining (8)-(11) and summarized as,

$$
F_{c}=g\left(h, x_{s d}\right) \text {. }
$$

Finally, the theoretical model about the pulling force on the cable and the input air pressure inside the soft actuator as displacement of the slider increases can be obtained by combining (12) and (7).

Of note, the distance between the soft linear actuator and the two cables will aslo influence the bending performance of the soft actuator. Preliminary theoretical analysis shows that the maximum bending angle of the soft actuator happens when cable \#1 gets its maximum length and cable \#2 is totally locked by clutch \#2 without any elongation in length. Considering the fact that the maximum locking force on the cables is limited, which means we have to enlarge the moment arm of cable \#2 as much as possible to lock it within a limited locking force. However, the experimental result shows that cable \#1 will interfere with the soft actuator when bending if they are mounted too close to each other as shown in Figure 1. Hence, the distances between the soft actuator and the two cables are determined finally by combining the theoretical and experimental results to achieve the maximum bending angle of the soft actuator with limited locking force and stroke on the cables.

\subsection{Soft modular legs}

Adaptability for various working scenarios and enough holding force to support the robot against gravity are the main requirements in the design of legs for climbing robots. For the former, three types of modular designed legs are introduced in this work as illustrated in Figure 4, which could significantly improve the adaptability and meanwhile offer new insights into designing climbing robots. For the latter, a general method to enhance the gripping force when holding objects for existing totally-soft grippers is to apply higher input air pressure. However, insufficient contact between the soft actuators and objects happens sometimes and a gripping failure will be induced by excessive deformation of the soft structures when overlarge input air pressure is applied, as mentioned in $[25,26]$. Trying to offer an alternative solution for this general problem of totally-soft actuators, a soft-rigid hybrid structure is hereby proposed based on a similar design method with the work in [30]. As validated in [30], the gripping force can be significantly enhanced by the bulged out 
TABLE I. Coefficients of Soft Materials

\begin{tabular}{|c|c|c|c|}
\hline \multirow{2}{*}{ Materials } & \multicolumn{3}{|c|}{ Coefficients (MPa) } \\
\cline { 2 - 4 } & $\mathrm{C} 1$ & $\mathrm{C} 2$ & $\mathrm{C} 3$ \\
\hline Elastosil M4601 & 0.1 & $1.2 \times 10-2$ & $7.5 \times 10-4$ \\
\hline $\begin{array}{c}\text { Dragon Skin 30 with } \\
10 \% \text { Silicone Thinner }\end{array}$ & $5.1 \times 10-2$ & $1.8 \times 10-2$ & $-9.2 \times 10-4$ \\
\hline
\end{tabular}

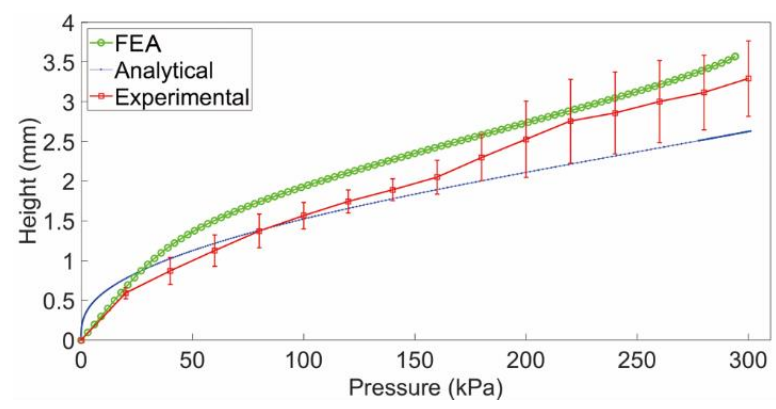

Figure 5. FEA, Analytical and experimental results of the height of the silicone bulges of the soft rectangular actuator inside the origami bellow as the input air pressure increases.

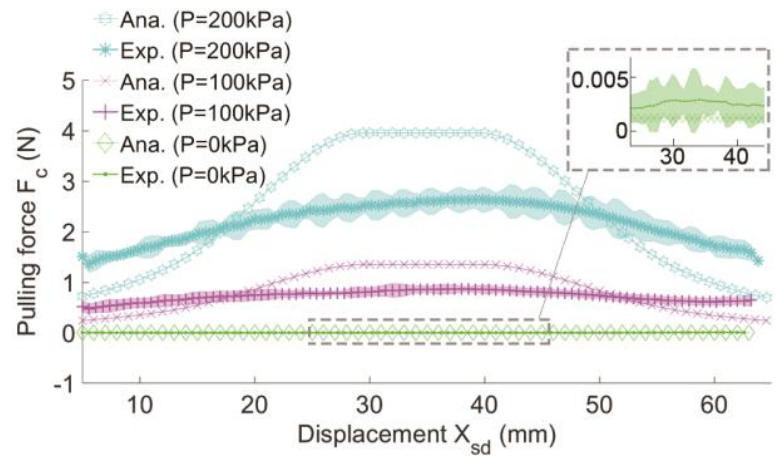

Figure 6. Analytical and experimental results of the relationship between the pulling force on the cable in the origami clutch and the displacement of the slider when the input air pressure inside the soft actuator of the origami bellow is set at $0 \mathrm{kPa}, 100 \mathrm{kPa}$ and $200 \mathrm{kPa}$ respectively.

silicone bubbles. In this paper, each of the three types of legs are made of a soft rectangular actuator and a different shell to restrict undesired deformation of the actuator. Identical soft actuators are utilized in this work to simplify the design and fabrication process.

For pipelines with varied diameters and bends, a normally open leg-type named as type \#1 in Figure 4 is proposed. The multi-segment origami shell is laminated by four laser-cut layers, including two identical multi-segment fiberglass layers as the main support structure, a Kapton and adhesive layer for linkage and attachment of fiberglass layers, and a TPU layer with creases to avoid detachment by restricting the maximum elongation of the dorsal side of the leg when bending. It must be noted that similar design about the holes in the fiberglass layer of the origami bellow as mentioned above is also applied here so that silicone can bulge out from the holes to directly contact the pipes. Since the friction factor between silicone and the material of pipes is higher than the one between fiberglass and pipes, the gripping force can be relatively enhanced with this design. Preliminary experimental results showed that the climbing robot tended to slide down the pipe when normally totally-soft bending actuators were adopted due to detachment between the soft actuators and pipes as described in $[25,26]$. In addition, if there were no holes on the origami shells, failure also happened even if the origami shellbased soft-rigid hybrid actuators were utilized, since the friction factor between the shell and pipes is relatively low. Finally, the robot was able to climb up pipes with any inclined angles carrying a certain load with the help of TPU layer to avoid excessive bending of the actuators and silicone bulges to enhance gripping force. The detailed results will be given in the following section.

To fulfil the requirements in certain circumstances where large load ability is needed while climbing along the outside of pipes, a normally closed leg-type is proposed as denoted as type \#2. The shell for this type is 3D printed with several holes on the inner side. When inflated, silicone bulges from the holes contacts pipes and a larger gripping force can be achieved since the rigid shells are totally fixed. Hence, the robot is able to climb up a pipe of a certain diameter with a large external load. Similarly, a normally closed leg-type (type \#3) is proposed for in-pipe climbing robots. The biggest difference between this leg-type and the former-mentioned type is that the holes are designed on the outer side of the 3Dprinted shell so that the silicone bulges can interact with the inner side of the pipes to hold the robot. Schematic of the possible application of the three modes for the pipe-climbing robot in various working scenarios are given in Figure 4.

\section{Results}

To verify the analytical models of the proposed origami clutches, several groups of experiments were conducted. Then, analytical results were compared with simulational and experimental ones as illustrated in Section 3.1. In addition, the workspace of the pipe-climbing robot was tested in Section 3.2 to quantitively evaluate its multi-DoF motion ability with the help of the proposed origami clutches. Finally, climbing and loading capacity tests were conducted on a prototyped pipe-climbing robot and results can be found in Section 3.3.

\subsection{Verification of theoretical models}

Standard uniaxial extension tests of the utilized soft materials were conducted to obtain their material coefficients before simulation and experimental verification of the analytical models,. The detailed material coefficients based on Yeoh model are given in Table I.

First part was the verification of the theoretical model between the deformation of the soft rectangular actuator inside 


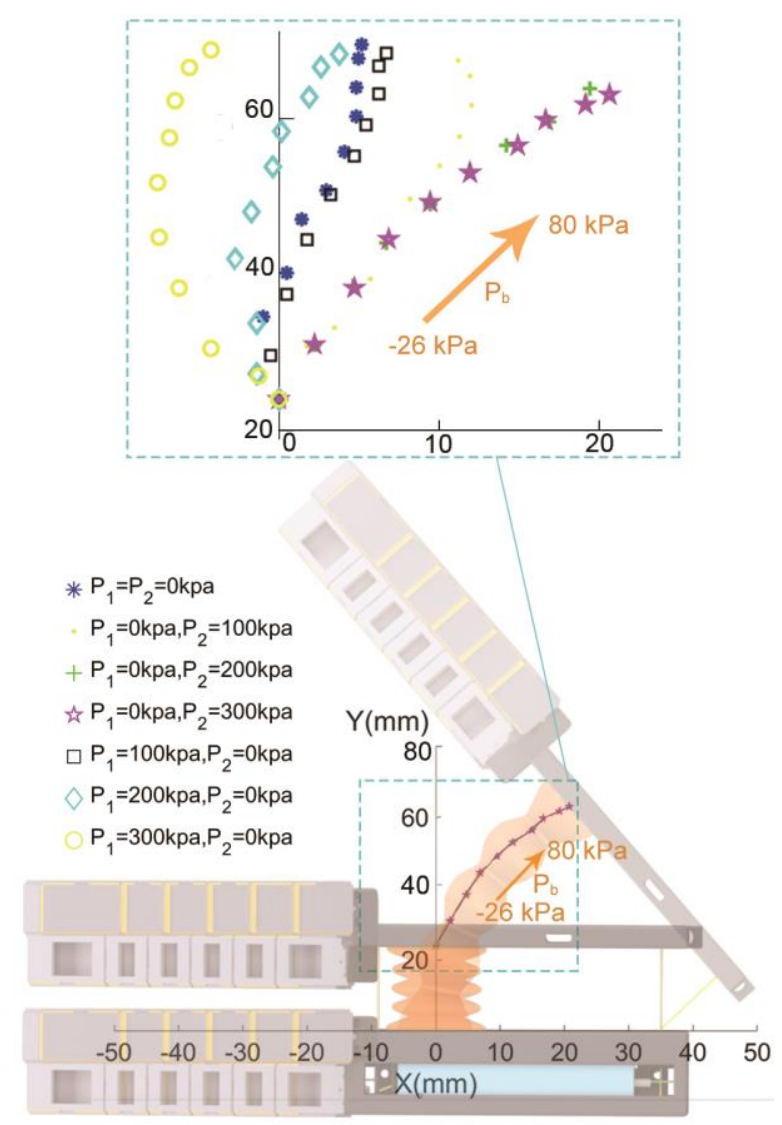

Figure 7. Workspace of the pipe climbing robot in out-pipe versatile mode. Closeup view: the moving trajectories of the soft bellow by choosing the center on the top surface of the soft linear actuator as the reference point when the actuator is vacuumed or inflated from -26 $\mathrm{kPa}$ to $80 \mathrm{kPa}$ and the input pressure in the two origami clutches varies from $0 \mathrm{kPa}$ to $300 \mathrm{kPa}$ with a $100 \mathrm{kPa}$ gap.

the origami bellow and the input air pressure. Both FEM simulation and experiments were conducted.

Since the soft rectangular actuator is totally symmetric in longitudinal and cross-sectional directions, only a quarter of the soft actuator was simulated to save computation costs. The boundary conditions utilized here are identical with the reality. Simulation results showed that the membrane of the soft actuator at the holes will be inflated to a spherical shape, and the local stress at each element of spherical-shaped silicone bulges is almost the same except at the tip area with higher stress, which means that the assumption in the theoretical models is rational. It should be noted that the small difference in stress at the tip area might induce a little deviation between the simulation and calculational results because the Eq. (4) has implied an assumption that the stress is equally distributed over all the spherical areas. Then, the height of silicone bulges in the FEA results was extracted for further comparison with the analytical models.

In the experimental part, tests on three separately fabricated soft actuators were conducted to obtain their relationship between the height of the silicone bulges and input air pressure. To eliminate the random error, each experiment for each sample was repeated three times. The final FEA, analytical and experimental results of the deformed height of the silicone bulges of the soft rectangular actuator as the input pressure increases are given in Figure 5. As can be seen, the deviation is acceptable within the error margin.

Then, we conducted experiments on a homemade platform to verify the analytical model between the pulling force on the cable and the displacement of the slider for any certain input pressure inside the origami bellow. During the experiments, the slider was pulled by a load cell to move along the slideway when the input air pressure was set at $0 \mathrm{kpa}, 100 \mathrm{kPa}$ and 200 $\mathrm{kPa}$ respectively, and the pulling force was recorded. Each experiment was also repeated three times to eliminate random error. Elastic strings as shown in Figure 1 were removed in these experiments for a more intuitive comparison of the experimental and analytical results. Besides, it should be noted that the friction coefficients between the slider and the bottom plate of the origami bellow shell as well as the slideway as mentioned above were also calibrated with similar platforms and the results are $\mu_{s}=0.12, \mu_{b}=0.71$.

Finally, analytical and experimental results are given in Figure 6. As illustrated, similar variation trends were found between the analytical and experimental curves when the displacement of the slider increased under each input air pressure. However, relatively large deviation also exists for the results, especially for higher air pressure. A possible main reason could be due to propagated error coming from the deviation in the deformed height. In addition, fabrication error of the 3D-printed platform to mount the origami bellow and the slideway might also contribute. The plane to attach the origami bellow is difficult to keep totally even due to the limited precision of the 3D printer and any tiny uneven spot might induce large deviations in the final results because the pulling force is quite sensitive for the distance between the bottom bellow plate and the slider according to the analytical model.

\subsection{Workspace of the pipe-climbing robot}

As mentioned above, the soft linear actuator will bend towards different directions when inflated as input pressure in the two origami clutches varies. To quantitively validate the multi-DoF motion ability of the soft linear actuator based on the proposed pneumatic origami clutches, the workspace of the body of the pipe-climbing robot was analyzed. Only outpipe versatile mode was adopted here for simplification.

During the experiments, the soft linear actuator was vacuumed or inflated from $-26 \mathrm{kPa}$ to $80 \mathrm{kPa}$ with the input pressure in the two clutches from $0 \mathrm{kPa}$ to $300 \mathrm{kPa}$ respectively with a 100 $\mathrm{kPa}$ gap. The center on the top surface of the soft linear actuator was selected as the reference point and its trajectories when the actuator moves were recorded as shown in Figure 7. The results showed that a maximum $289.6 \%$ linear stroke on 


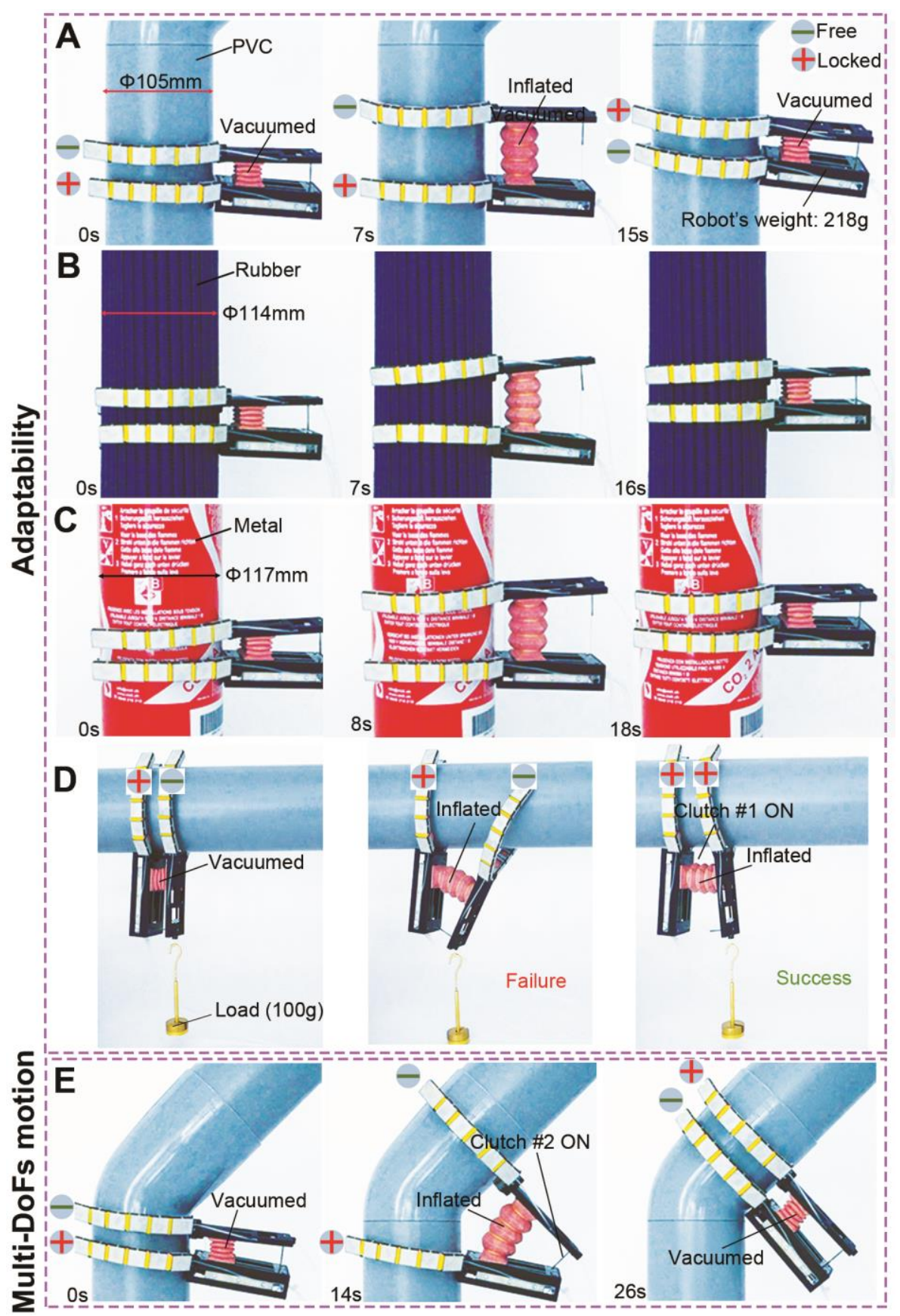

Figure 8. Demonstration of the pipe-climbing robot in out-pipe versatile mode. (A-C) Climbing on the exterior of vertical pipes made of PVC, rubber and metal respectively with diameters ranging from 105 $\mathrm{mm}$ to $117 \mathrm{~mm}$. (D) Navigating the exterior of a horizontal pipe: with both origami clutches unactuated, failure will occur due to unexpected bending of the actuator induced by the external load and unbalanced bending moment caused by the two cables; by adjusting the input air pressure inside clutch \#1, the corresponding cable \#1 will be selectively tightened to balance the bending moment so that the climber can maintain functional. (E) Traveling along the outside of pipes with 45 degrees bends.

the soft linear actuator and maximum 45 degrees bending angle could be achieved by selectively adjusting the input air pressure inside the two pneumatic origami clutches.

\subsection{Prototype evaluation}

A prototype multimodal pipe-climbing robot was also developed in this work to further demonstrate the effectiveness of the proposed pneumatic origami clutches and soft modular legs. As introduced above, three climbing modes of the robot can be achieved by simply replacing leg-types for different working scenarios.

For out-pipe versatile mode, two pairs of normally open 


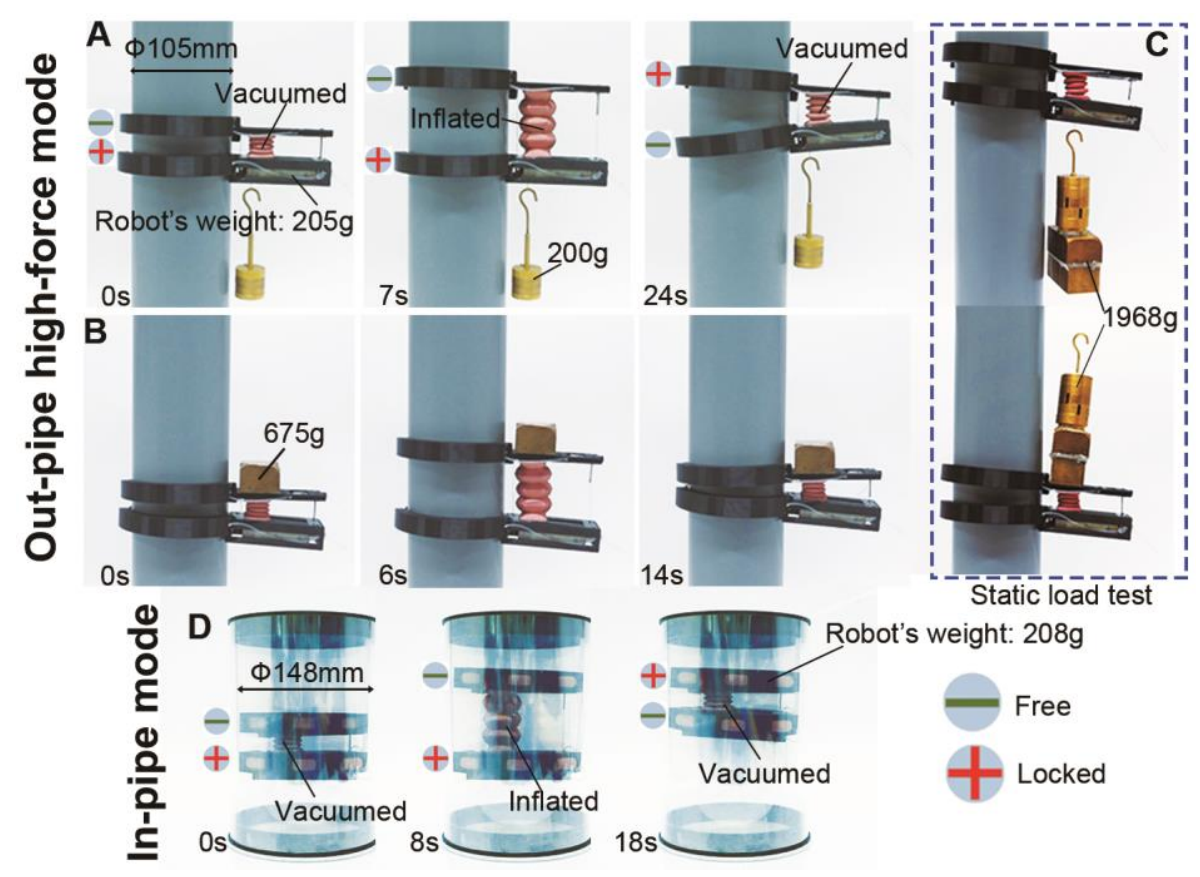

Figure 9. Demonstration of the pipe-climbing robot in out-pipe high-force mode and in in-pipe mode. Outpipe high-force mode: (A) and (B) climbing along the outside of a PVC pipe with $200 \mathrm{~g}$ payload hanging from the bottom of the climber or $675 \mathrm{~g}$ payload attached at the top; (C) payloads as heavy as $1968 \mathrm{~g}$ can be sustained without failure for this mode. In-pipe mode: (D) the climber can navigate inside of pipes with diameters around $148 \mathrm{~mm}$.

legs as shown in Figure 4 were installed on the main body of the climbing robot. As illustrated in Figure 8A-C, experimental results showed that the robot can climb up pipes of different materials (PVC, rubber and metal) and varying outer diameters ranging from 105 to $117 \mathrm{~mm}$ by periodic actuation of the soft linear actuator and legs. Besides, when navigating along a horizontal pipe carrying an external load as illustrated in Figure 8D, the robot tended to fail due to the influence of its own weight and the external load with no clutch actuated. However, the climbing robot was then able to keep climbing by selectively adjusting the input air pressure in clutch \#1 to restrict the undesired deformation of the soft linear actuator, which is another advantage of the proposed origami clutches over totally-soft actuators. Furthermore, the robot could also deal with pipes with bends of up to 45 degrees by actuating the origami clutch \#2 as shown in Figure $8 \mathrm{E}$. Demonstration videos showing the pipe-climbing robot in outpipe versatile mode can be found in Supplementary Video S1S2.

For out-pipe high-force mode, the climbing robot consists of a main body and two pairs of normally closed legs with holes at the inner side of the 3D-printed shell as shown in Figure 4. In this mode, the robot was able to climb up vertical pipes of certain outer diameter with a payload of up to $200 \mathrm{~g}$ hanging from the bottom or $675 \mathrm{~g}$ mounted on the top as illustrated in Figure 9A and 9B. Besides, a maximum 1968g payload, 9.6 times the weight of the robot ( $205 \mathrm{~g}$ in this mode), could be sustained by the climbing robot in static working mode (no climbing) according to the experimental results as shown in Figure 9C, which could be helpful in certain circumstances. Of note, the loading will also affect the performance of the robot and reduce the stroke of the soft linear actuator. When the load was hung from the bottom of the robot as in Figure 9A, the push-up process will not be influenced, while slower contraction speed of the soft linear actuator was observed in the recovering process. Besides, the soft actuator would fail to recover when too much payload was applied. When the load is mounted on the top of the robot as in Figure 9B, a slower elongation speed and a smaller stroke of the soft actuator was observed in the push-up process due to the influence of the payload, while the recovering process was not affected. Corresponding demonstration video can be found in Supplementary Video S3.

For in-pipe mode, the main body of the robot was integrated with two pairs of normally closed legs with holes at the outer side of the 3D-printed shell. When inflated, the silicone bulges popped up from the holes held the robot against the inside of the pipe. Hence, by selectively actuating the soft linear actuators and legs, the robot was able to navigate inside a pipe of $148 \mathrm{~mm}$ inner diameter as illustrated in Figure 9D. Related demonstration video can be found in Supplementary Video S4.

\section{Conclusion and discussion}

In this paper, we present a new design of a multimodal pipe-climbing robot with origami clutches and soft modular 
legs to try to solve the two main challenges in bioinspired pipe-climbing robots: a versatile body for large-stroke propulsion and multi-DoF motion, and adaptable legs to exert enough holding force in several working scenarios. Firstly, a novel pneumatic origami clutch consisting of a soft actuatorbased origami bellow and a slider-slideway mechanism is proposed for soft structures to realize multi-DoF motion. Analytical models about the deformation of the soft actuator inside the pneumatic origami bellow and the pulling force on the cable connected to the slider when input air pressure varies are introduced in detail and then compared to FEA and experimental results. Preliminary results show that a stroke up to $289.6 \%$ and a maximum 45 degrees-bending on the soft linear actuator can be achieved by selectively adjusting the input air pressure inside the origami clutches. Then, three types of soft modular legs are proposed for multimodal climbing of the robot in various working scenarios. Experimental results show that this design not only avoids grasping failure caused by detachment between the normally open legs and pipes when excessive input air pressure is applied, but also enhances the grasping force for the normally closed legs. To evaluate the performance of the origami clutches and soft modular legs, a prototype pipe-climbing robot is further developed. Experimental results show that (1) in the out-pipe versatile mode, the robot is suitable for pipes made of various materials including PVC, rubber and metal with diameters ranging from 105 to $117 \mathrm{~mm}$ by periodic actuation of the soft linear actuator and legs. Besides, the robot is also able to deal with pipes with bends of up to 45 degrees by selectively regulating the input air pressure inside the origami clutches. Furthermore, the prototype proposed in this work can also avoid failure due to the carried external load and the inherent low stiffness of the soft linear actuator when navigating outside a horizontal or inclined pipe, which might be difficult for existing multi-chamber soft pipe climbing robots; (2) In the out-pipe high-force mode, navigating along a specific pipe with max imum $675 \mathrm{~g}$ external load at the top or $200 \mathrm{~g}$ hanging from the bottom, and keeping functional without failure under static load as high as $1968 \mathrm{~g}$ can be achieved; (3) In the in-pipe mode, the robot is then capable of traveling inside pipes with $148 \mathrm{~mm}$ inner diameter. This research might not only offer a new way for soft robots to realize multi-DoF motion and enhance their grasping force without weakening the inherent compliance of soft materials, but also bridges the design gap between in-pipe and out-pipe climbing robots.

In the future, we will focus on optimizing and simplifying the origami clutches to selectively adjust the bending angles as well as the structural stiffness of soft actuators. In addition, pipe-climbing robots which can navigate in a larger workspace and negotiate better various obstacles will also be studied.

\section{Acknowledgements}

This research is supported by the China Scholarship Council, National Natural Science Foundation of China under grant 51775012 and the Beijing Municipal Science \& Technology Project under grant Z181100003118005. The authors would also like to express their great gratitude to Dr. Huijuan Feng for the analytical models and Mr. Z. Zhakypov for helpful discussion on the structure design.

\section{References}

[1] Chattopadhyay, P., Ghoshal, S., Majumder, A. and Dikshit, H., 2018. Locomotion Methods of Pipe Climbing robots: A Review. Journal of Engineering Science \& Technology Review, 11(4).

[2] Schmidt, D. and Berns, K., 2013. Climbing robots for maintenance and inspections of vertical structures-A survey of design aspects and technologies. Robotics and Autonomous Systems, 61(12), pp.1288-1305.

[3] Neubauer, W., 1994, September. A spider-like robot that climbs vertically in ducts or pipes. In Proceedings of IEEE/RSJ International Conference on Intelligent Robots and Systems (IROS'94) (Vol. 2, pp. 1178-1185). IEEE.

[4] Haynes, G.C., Khripin, A., Lynch, G., Amory, J., Saunders, A., Rizzi, A.A. and Koditschek, D.E., 2009, May. Rapid pole climbing with a quadrupedal robot. In 2009 IEEE International Conference on Robotics and Automation (pp. 2767-2772). IEEE.

[5] Waleed, D., Mustafa, S.H., Mukhopadhyay, S., Abdel-Hafez, M.F., Jaradat, M.A.K., Dias, K.R., Arif, F. and Ahmed, J.I., 2018. An In-Pipe Leak Detection Robot with a NeuralNetwork-Based Leak Verification System. IEEE Sensors Journal, 19(3), pp.1153-1165.

[6] Kakogawa, A., Nishimura, T. and Ma, S., 2016. Designing arm length of a screw drive in-pipe robot for climbing vertically positioned bent pipes. Robotica, 34(2), pp.306-327.

[7] Xu, F., Jiang, Q., Lv, F., Wu, M. and Zhang, L., 2018. The Dynamic Coupling Analysis for All-Wheel-Drive Climbing Robot Based on Safety Recovery Mechanism Model. Applied Sciences, 8(11), p.2123.

[8] Sarvestani, A.A., Eghtesad, M., Fazlollahi, F., Goshtasbi, A. and Mokhtari, K., 2016. Dynamic modeling of an out-pipe inspection robot and experimental validation of the proposed model using image processing technique. Iranian Journal of Science and Technology, Transactions of Mechanical Engineering, 40(1), pp.77-85.

[9] Fang, H., Zhang, Y. and Wang, K.W., 2017. Origami-based earthworm-like locomotion robots. Bioinspiration \& biomimetics, 12(6), p.065003.

[10]Yamamoto, T., Konyo, M., Tadakuma, K. and Tadokoro, S., 2018. High-speed sliding-inchworm motion mechanism with expansion-type pneumatic hollow-shaft actuators for in-pipe inspections. Mechatronics, 56, pp.101-114.

[11]Takeshima, H. and Takayama, T., 2018. Geometric estimation of the deformation and the design method for developing helical bundled-tube locomotive devices. IEEE/ASME Transactions on Mechatronics, 23(1), pp.223-232.

[12]Rus, D. and Tolley, M.T., 2015. Design, fabrication and control of soft robots. Nature, 521(7553), p.467. 
[13]Verma, M.S., Ainla, A., Yang, D., Harburg, D. and Whitesides, G.M., 2018. A soft tube-climbing robot. Soft robotics, 5(2), pp. 133-137.

[14]Adams, W., Sridar, S., Thalman, C.M., Copenhaver, B., Elsaad, H. and Polygerinos, P., 2018, April. Water pipe robot utilizing soft inflatable actuators. In 2018 IEEE International Conference on Soft Robotics (RoboSoft) (pp. 321-326). IEEE.

[15]Zhou, J., Yi, J., Chen, X., Liu, Z. and Wang, Z., 2018. BCL-13: A 13-DOF Soft robotic hand for dexterous grasping and in-hand manipulation. IEEE Robotics and Automation Letters, 3(4), pp.3379-3386.

[16]Bruder, D., Remy, C.D. and Vasudevan, R., 2019, May. Nonlinear system identification of soft robot dynamics using koopman operator theory. In 2019 International Conference on Robotics and Automation (ICRA) (pp. 6244-6250). IEEE.

[17]She, Y., Chen, J., Shi, H. and Su, H.J., 2016. Modeling and validation of a novel bending actuator for soft robotics applications. Soft Robotics, 3(2), pp.71-81.

[18]Schubert, B.E. and Floreano, D., 2013. Variable stiffness material based on rigid low-melting-point-alloy microstructures embedded in soft poly (dimethylsiloxane)(PDMS). Rsc Advances, 3(46), pp.24671-24679.

[19]Singh, G., Patiballa, S., Zhang, X. and Krishnan, G., 2019, May. A Pipe-Climbing Soft Robot. In 2019 International Conference on Robotics and Automation (ICRA) (pp. 84508456). IEEE.

[20]Zhang Z., Wang X., Wang S., Meng D., and Liang B., 2019. Design and modeling of a parallel-pipe-crawling pneumatic soft robot. IEEE Access, 7, pp. 134301-134317.

[21]Hauser, S., Robertson, M., Ijspeert, A. and Paik, J., 2017. Jammjoint: A variable stiffness device based on granular jamming for wearable joint support. IEEE Robotics and Automation Letters, 2(2), pp.849-855.

[22]Kim, Y.J., Cheng, S., Kim, S. and Iagnemma, K., 2013. A novel layer jamming mechanism with tunable stiffness capability for minimally invasive surgery. IEEE Transactions on Robotics, 29(4), pp.1031-1042.

[23]Firouzeh, A., Salerno, M. and Paik, J., 2017. Stiffness control with shape memory polymer in underactuated robotic origamis. IEEE Transactions on Robotics, 33(4), pp.765-777.

[24]Han, S., Ahn, J. and Moon, H., 2016. Remotely controlled prehensile locomotion of a two-module 3D pipe-climbing robot Journal of Mechanical Science and Technology, 30(4), pp.18751882 .

[25]Glick, P., Suresh, S.A., Ruffatto, D., Cutkosky, M., Tolley, M.T. and Parness, A., 2018. A soft robotic gripper with geckoinspired adhesive. IEEE Robotics and Automation Letters, 3(2), pp.903-910.

[26]Hirose, S. and Umetani, Y., 1978. The development of soft gripper for the versatile robot hand. Mechanism and machine theory, 13(3), pp.351-359.

[27]Lee, J.G. and Rodrigue, H., 2019. Origami-Based Vacuum Pneumatic Artificial Muscles with Large Contraction Ratios. Soft robotics, 6(1), pp.109-117.

[28]Zhakypov, Z., Mete, M., Fiorentino, J. and Paik, J., 2019, April. Programmable Fluidic Networks Design for Robotic Origami Sequential Self-Folding. In 2019 2nd IEEE International Conference on Soft Robotics (RoboSoft) (pp. 814-820). IEEE

[29]Popov, V.L., 2013. Method of reduction of dimensionality in contact and friction mechanics: A linkage between micro and macro scales. Friction, 1(1), pp.41-62.

[30]Becker, K.P., Bartlett, N.W., Malley, M.J., Kjeer, P.M. and Wood, R.J., 2017, May. Tunable friction through constrained inflation of an elastomeric membrane. In 2017 IEEE International Conference on Robotics and Automation (ICRA) (pp. 4352-4357). IEEE. 